科 学通 报

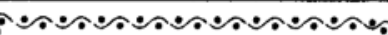

研究简报

\title{
关于事实反驳与一般假说的开放逻辑
}

\author{
苏开乐 丁德成
}

(南京大学数学系, 南京 210008)

\section{关链词 假说、开放逻辑、理想事实反驭}

最近李末 ${ }^{[1]}$ 给出了一个可以刻画知识的增长、更新以及假说的进化的开放逻辑理论. 开放逻 辑的一个重要概念就是假说重构. 开放逻辑的其它重要概念如认识进程等的定义及其有关性质 都可依赖于假说重构的概念而得到. 假说重构有两种: $N$-重构和 $R$-重构, 从构造上说, $N$-重构较 为简单, $R$-重构较为复杂. 故 $R$-重构可以说是建立开放逻辑的关键. 而 $R$-重构相应的模型论概 念是理想事实反驳, 因而对事实反驳的进一步研究无论对开放逻辑本身, 对开放逻辑在知 识库和专家系统中的应用, 对新的开放逻辑建立都有重要意义. 对于一给定的假说 $\Gamma$, 本文明 确给出了事实反驳的优先序关系, 而把理想事实反驳的概念作为上述优先序关系的极小元 而得到. 由以上优先序关系又能得到一等价关系, 从而很自然地把理想事实反驳的等价类与 $R$ - 重构一一对应起来.

开放逻辑中的假说的公式要求为闭的, 本文讨论了如何对一般的假说 (即其中公式可以含 有自由变元) 建立理想事实反驳, $R$-重构的概念, 从而仿照文献[1]中的办法建立关于一般假说 的开放逻辑. 认为有必要建立恰当的一般假说的开放逻辑, 正如有必要建立恰当开缺省逻辑一 样. Reiter ${ }^{[2]}$ 早就意识到开缺省的问题, 但他把其中的自由个体变元仅看作为基项的元变元是不 能满意的. 到近来缺省逻辑的语义学发展之后, Lifschitz ${ }^{[3]}$ 才建立了较令人满意的开缺省逻辑.

考虑含有谓词 $P$ 和不同的基项 $c_{n} c_{1} \cdots, c_{n}$ 的语言 $L$ 上的假说 $\Gamma=\{P(x)\}$, 显然 $\Gamma=\mathrm{P}\left(C_{0}\right)$, 问题: $P\left(c_{o}\right)$ 受到事实反驳时, 如何给出 $\Gamma$ 关于 $P\left(c_{o}\right)$ 的事实反驳的 $R$ - 重构?

若为了应用文献 [1]中关于闭假说的开放逻辑理论, 将假说中公式的自由变元加上量词封闭而 得到假说 $\Gamma_{1}=\{(x) p(x)\} . \Gamma_{1}$ 关于 $p\left(c_{0}\right)$ 的事实反驳的 $R$-重构为 $\left\{\neg P\left(c_{o}\right)\right\}$. 但若把 $\Gamma$ 关 $P\left(c_{0}\right)$ 的 $R-$ 重构给出为 $\left\{\neg p\left(c_{o}\right)\right\}$ 是与直觉不符的. $R$-重构的直觉意义是当假说中推出的某结论受到反驳 时, 我们只修改那些在假说中导致此结论的最小的假设集. $\Gamma$ 受到关于 $p\left(c_{o}\right)$ 的事实反驳时, $\Gamma$ 中原有的 $p\left(c_{1}\right) \cdots\left(c_{n}\right)$ 不应被删去. 直觉上 $\Gamma$ 关于 $p\left(q_{b}\right)$ 的 $R$ - 重构应为 $\left\{\neg p\left(c_{o}\right), x \neq c_{o} \rightarrow p(x)\right\}$.

若把假说 $\Gamma$ 等同于假说 $\Gamma_{2}=\left\{p\left(c_{0}\right), p\left(c_{1}\right), \cdots, p\left(c_{n}\right)\right\}$, 即把 $p(x)$ 中的变元 $x$ 等同于关于基项 $c_{0}$, $\cdots, c_{n}$ 的元变元, 但 $\Gamma_{2}$ 关于 $p\left(c_{0}\right)$ 的事实反驳的 $R$ - 重构为 $\left\{7 p\left(c_{0}\right), p\left(c_{1}\right) \cdots p\left(c_{n}\right)\right\}$, 它比直觉上 $\Gamma$ 关于 $P\left(c_{0}\right)$ 的事实反驭的-重构 $\left\{\neg p\left(c_{0}\right), x \neq c_{0} \rightarrow p(x)\right\}$ 要弱, 除非我们接受所谓封闭世界假设:

$$
\text { (x) }\left(x=c_{0} \vee x=c_{1} \vee \cdots x=C_{n}\right) \text {, }
$$

$1993-09-28$ 收稿. 
封闭世界假设有时是不能接受的, 我们常常不能也不愿设计这样的语言使得推理领域内的每 一个个体都可以用一基项表示出来.

本文提出的关于一般假说的开放逻辑中, 假说中公式的自由变元作为真正的个体变元而 不是表示基项的元变元得到了恰当的处理. 我们从语义的模型论的概念事实反驳的讨论人 手, 因为借助于模型而讨论推理领域的个体可以很直接而方便, 而对于公式要涉及它们只好 通过它们的语法表示.

\section{1 事实反驳的优先序}

本节我们固定 $\Gamma$ 为开放逻辑中的一假说, 即一非空的相容的闭的公式集合, $A$ 为 $\Gamma$ 推出 的一结论. 我们称模型 $M$ 为 $A$ 的事实反驳当且仅当 $M \mid=7 A$.

定义 1 对 $A$ 的两个事实反驳 $M_{1}, M_{2}$, 我们说 $M_{1}$ 优先 $M_{2}$ (记为 $M_{1} \leqslant M_{2}$ ) 当且仅当对 任何 $\Gamma \Gamma \Gamma$ 公式 B 若 $M_{2} \vDash B$ 则 $M_{1} \models B$. 我们说 $M_{1}$ 等价于 $M_{2}$ ( 记为 $M_{1} \approx M_{2}$ ), 如果 $M_{1} \leqslant M_{2}$ 且 $M_{2} \leqslant M_{1}$.

定义 2 我们称 $M$ 为 $\Gamma$ 关于 $A$ 的理想事实反驳如果 $M$ 为 $A$ 的事实反驳且为上述 $\leqslant$ 关系的极小元即不存在另一个 $A$ 的事反驳 $M^{\prime}$ 使得 $M^{\prime} \leqslant M$ 且 $M^{\prime} \neq N^{\prime} M$.

我们指出定义 1 中 “ $\leqslant$ ”关系具有传递性, 因而 “ $\approx$ ”为一等价关系. 每个 $A$ 的事实反驳 $M$ 确定一个等价类记为 $\bar{M}$. 定义 2 中理想事实反驳的概念与文献[1] 中定义的理想事实反驳 是一致的.

定理 1 对任何 $\Gamma$ 的与 $7 A$ 相容的极大子集 $\Delta$, 令 $f(\Delta)=\{M \mid M$ 为 $A$ 的事实反驳 且驳且 $\mathrm{M}=\Delta\}$ 那么 $f$ 为 $\Gamma$ 的与 $\neg A$ 相容的极大子集的集合到 $A$ 的理想事实反驳的等价类 的集合的一一对应.

证 任给 $\triangle{ }_{1}, \triangle_{2}$ 为两个 $\Gamma$ 的与 $7 A$ 相容的极大子集, 由 $A$ 的理想事实反驳的定义知 $f\left(\triangle_{1}\right), f\left(\triangle_{2}\right)$ 都是 $A$ 的理想事实反驳的等价类, 且 $\triangle_{1} \neq \triangle_{2}$ 时有 $f\left(\triangle_{1}\right) \neq f\left(\triangle_{2}\right)$. 又 任 给 $A$ 的理想事实反驳的等价类 $\bar{M}$, 令 $\triangle=\{B \in \Gamma|M| \neq B\}$, 则 $\triangle$ 为 $\Gamma$ 的与 $\vDash A$ 相容的极大子集, 且有 $f(\triangle)=\bar{M}$. 证毕.

注 我们照以上方法也可给出其它不同的关于事实反驳的优先序关系, 从而新的形式 的开放逻辑就可建立. 例如给出 $\Gamma$ 中一个表示相信程度的良基偏序关系 $R$, 对事实反驭 $M_{1}, M_{2}$ 规定 $M_{1} \leqslant{ }^{\prime} M_{2}$ 当且仅当对任何 $M_{2}$ 中真而 $M_{1}$ 中 假的 $\Gamma$ 中的句子 $B$, 有一个 $M_{2}$ 中假而 $M_{1}$ 中 真的 $\Gamma$ 中的句子 $B^{\prime}$ 使得 $B^{\prime} R B$. 那么优先关系 $\leqslant^{\prime}$ 是一个自反的可传递的偏序, 新的形式的理 想事实反驳就可定义为以上优先关系的极小元, $R$ - 重构可由这种极小元确定, 这样可以建立 一种新开放逻辑.

\section{2 关于一般假说的 $R$ - 重构}

本节我们讨论如何对一般的假说建立开放逻辑理论, 我们仅给出理想事实反驳, $R$-重构 的定义, 其它重要概念如新假设重构, 认识进程可以参照文献[1] 而不难得到. 我们固定 $\Gamma^{*}$ 为一般的假说, 即一非空的相容的公式 (可含自由变元 $x$ ) 的集. $A$ 为 $\Gamma^{*}$ 推出的一句子. $U$ 为一可数无穷的个体域. 我们称模型 $M$ 为 $A$ 的 $U$-事实反驳, 如果 $M$ 的全域为 $U$ 的子集, 且 $M \vDash \neg A$. 
定义 3 对任何两个 $A$ 的 $U$-事实反驱 $M_{0}, M_{1}$, 我们说 $M_{1}$ 优先 $M_{0}$ (记为 $M_{1} \leqslant * M_{0}$ 或 $M_{0} \geqslant$ $\left.{ }^{*} M_{1}\right)$ 当且仅当 $\left|M_{0}\right| \subset\left|M_{1}\right|$, 且对任何 $\Gamma$ 中的公式 $B(x)$ 和任何个体 $\alpha \in\left|M_{0}\right|$, 若 $M_{0} \vDash B(a)$ 则 $M_{1} \models B(a)$. 我们说 $M_{0}$ 等价于 $M_{1}$, 如果 $M_{0} \leqslant * M_{1}$ 且 $M_{1} \leqslant * M_{0}$.

定义 4 我们称 $A$ 的 $U$-事实反驳 $M$ 为 $A$ 的 $U$ - 理想事实反驳, 如果 $M$. 为上述优先关 系的极小元, 即不存在 $A$ 的 $U$-事实反驭 $M^{\prime}$ 使得 $M^{\prime} \leqslant * M$ 且 $M^{\prime}$ 不等价于 $M$.

定义 3 中的 $\leqslant *$ 是反身的传递的序关系, 从而定义 3 给出的 “等价关系”确为一等价关 系. 当 $\Gamma^{*}$ 中的公式都为开公式时定义 4 中的极小元是存在的, 我们有

定理 2 当 $\Gamma^{*}$ 中的公式都为开公式时对任何 $A$ 的 $U$-事实反驳 $M_{0}$ 存在一个 $A$ 的 $U$ - 理想事实反驳 $M$ 使得 $M_{0} \geqslant * M$.

证 由 Zorn 引理只需证明对任何优先序 $\geqslant *$ 的 $U$ - 事实反驳的链 $M_{0} \geqslant * M_{1} \geqslant * \cdots M_{i} \geqslant * \cdots$, 有 $U$-事实反驳 $M$ 使得对任何 $i$ 有 $M_{i} \geqslant * M$. 取自然数集上的一个非主超滤子 $D$, 如下给出模 型 $M$, 首先 $M$ 的全域 $|M|=\left\{a \mid\left\{i|a \in| M_{i} \mid\right\} \in D\right\}$, 其对任何谓词 $P\left(x_{i} \cdots x_{n}\right)$ 和 $|M|$ 中的个体 $a_{1}, \cdots, a_{n}$, 规定 $P\left(a_{1}, \cdots, a_{n}\right)$ 在 $M$ 中真当且仅当 $\left\{i \mid M_{i} \vDash P\left(a_{1}, \cdots, a_{n}\right)\right\} \in D$. 用类似于超积模型的方法 ${ }^{(4)}$ 即可 证明 $M$ 为 $A$ 的 $U$ - 事实反驳且对任何 $i$ 有 $M_{i} \geqslant * M$. 证毕.

对给定的一个 $A$ 的 $U$ - 理想事实反驭 $M$, 我们如下给出一个 $\Gamma$ 关于 $M$ 的 $R$ - 重构 $R M$. 令 $T H(\bar{M})$ 为 $M$ 的等价类中每个模型都成立的句子集合, $R_{M}$ 可从原假说 $\Gamma^{*}$ 作出以下修改 而得到: (1) 把 $\neg A$ 加在 $\Gamma$ 中; (2) 删去使得 $T H(\bar{M}) \vdash(\forall x)(\neg B(x))$ 成立的 $\Gamma^{*}$ 中的公式 $B(x)$; (3) 保留使得 $T H(\bar{M}) \mid-(\forall) B(x)$ 成立的 $\Gamma^{*}$ 中的公式 $B(x)$; (4) 对不符合 (2), (3) 条件的 $\Gamma^{*}$ 中 的公式 $B(x)$ 改换成 $\vee S \rightarrow B(x)$ 形式的公式, 其中 $\vee S$ 表示 $S$ 中公式的析取, $S$ 表示使得 $T H$ $(\bar{M}) \vdash(x)(P(x) \rightarrow B(x))$ 成立的公式 $P(x)$ 的集合. 若 $\vee S$ 不能归约为一个公式 $Q(x)$, 我们则 把 $\vee S \rightarrow B(x)$ 看成 $P_{0}(x) \rightarrow B(x), \cdots, P_{i}(x) \rightarrow \pi(x), \cdots$ 等无穷个公式, 其中 $P_{i}(x) \in S$.

定义 $5 \Gamma^{*}$ 关于 $A$ 的事实反驳的 $R$ - 重构集为 $R$-recons $\left(\Gamma^{*}, A\right)=\left\{R_{M} \mid M\right.$ 为 $A$ 的 $U$ - 理 想事实反驳 $\}$.

例. $\Gamma^{*}=\{P(x)\}, A \equiv(x) P(x)$, 我们不难计算出 $\Gamma^{*}$ 关于 $A$ 的事实反驳的 $R$ - 重构仅有 一个为 $\{(\exists x)(\neg P(x)), \exists y(\neg P(y)) \& x \neq y \rightarrow P(x)\}$.

最后我们得出当 $\Gamma^{*}$ 中的公式皆为闭公式时以上 $R$-重构的定义与文献 [1] 的是完全一 致的.

致谢本文作者感谢导师莫绍揆先生的指导, 感谢李未教授把我们带进开放逻辑这一极 有理论研究和实际应用价值的领域.

\section{参考文甫}

[1] Li Wei, Science in China, Series A, 1993,36 (3): 362- 375.

[2] Reiter, R., Artif. Intell., 1980, 13: 81-132.

[ 3] Lifschitz, V., in Computational Logic, Springer-Verlag, Berlin, 1990.

[4] Chang, C., Keisler, J., Model Theory, North-Holland, Amsterdam, 1970. 